\title{
AODV-PA: AODV with Path Accumulation
}

\author{
Sumit Gwalani Elizabeth M. Belding-Royer \\ Department of Computer Science \\ University of California, Santa Barbara \\ \{sumitg, ebelding\}@cs.ucsb.edu
}

\author{
Charles E. Perkins \\ Communications System Laboratory \\ Nokia Research Center \\ charliep@iprg.nokia.com
}

\begin{abstract}
Ad hoc networks are the solution to the demands of spontaneous network setup. They are characterized by the use of wireless links, dynamically changing topology, multi-hop connectivity and decentralized routing mechanisms and decision-making. AODV and DSR are the two most widely studied on-demand ad hoc routing protocols. Previous studies have shown limitations of these protocols in certain network scenarios. To improve the performance of AODV, we modify AODV to include the source route accumulation feature of DSR. We call this AODV with path accumulation. This protocol optimizes AODV to perform effectively in terms of routing overhead and delay during high load. The performance of the protocol is evaluated by a simulation model under a variety of network conditions. We also compare its performance with that of unmodified AODV and DSR. We demonstrate how a small change to the AODV protocol can lead to significantly improved performance results.
\end{abstract}

\section{INTRODUCTION}

An ad hoc network is a collection of mobile nodes that are capable of communicating with each other without the aid of any established infrastructure or centralized administration. They are self-organized, dynamically changing multi-hop networks. Each node in an ad hoc network performs the dual task of being a possible source/destination of some packets while at the same time acting as a router for other packets to their final destination. In recent years, a variety of new routing protocols targeted specifically at this environment have been developed and studied.

The design issues of multi-hop ad hoc network protocols include the ability of the protocol to perform optimally under a wide variety of conditions. Two of the leading ad hoc network routing protocols are the Ad hoc On-Demand Distance Vector Routing protocol (AODV) [1] and the Dynamic Source Routing protocol (DSR) [2]. Previous work [3] has studied the performance of AODV and DSR in a variety of scenarios. This work showed that both AODV and DSR drop in performance at high velocities or when the number of connections is high. Based on the results, the authors proposed modifications to both AODV and DSR that could improve the performance of each protocol. One specific proposal is the accumulation of the source route in request and reply packets during the route discovery process in AODV. By accumulating this information, nodes can learn an increased amount of routing information to different destinations. Because of the resulting decrease in the number of route discoveries, the proposed modification should lead to a reduction in the routing load of AODV.

This paper studies the proposed modifications by incorporating the path accumulation feature in AODV. The conservative nature of our protocol helps to decrease the routing load, while at the same time maintaining good performance in application oriented metrics such as delay. To evaluate the new protocol, we present a detailed packet-level simulation comparing the performance of AODV with path accumulation to AODV and DSR.

\section{OVERVIEW OF AODV AND DSR}

\section{A. $A O D V$}

AODV is an on-demand dynamic routing protocol that uses routing tables with one entry per destination. When a source node needs a route to a destination, it initiates a route discovery process to locate the destination node. The source node floods a query packet requesting a route to be set up to the destination. A reply is sent back directly to the source node either by the destination itself or any other intermediate node that has a current route to the destination. On receiving a route request (RREQ), intermediate nodes update their routing table for a reverse route to the source. Similarly, the forward route to the destination is updated on receiving a route reply (RREP) packet. AODV uses sequence numbers to determine the timeliness of each packet and to prevent loops. Expiry timers are used to keep the route entries fresh.

Link failures are propagated by a route error (RERR) message from the site of a link break to the source node for that route. When the next hop link breaks, RERR packets are sent to a set of neighboring nodes that communicate over the broken link with the destination. This recursive process erases all broken entries in the routing table of the nodes. Since nodes reply to the first arriving RREQ, AODV favors the least congested route instead of the shortest route. The AODV on-demand approach minimizes routing table information. However, this potentially leads to a large number of route requests being generated [3].

\section{B. DSR}

Dynamic Source Routing (DSR) utilizes source-based routing rather than table-based. DSR is also an on-demand protocol and has a similar route discovery process to AODV. One of the primary differences between DSR and AODV is that intermediate node addresses are accumulated on the DSR RREQ and RREP control packets. Every node in the network uses the information in the RREQ/RREP packets to learn about routes to other nodes in the network. These nodes store the routes in their route caches.

Once a RREP is received, the sender node knows the entire route to the destination. Data packets in DSR are routed by the intermediate nodes using the complete route to the destination 


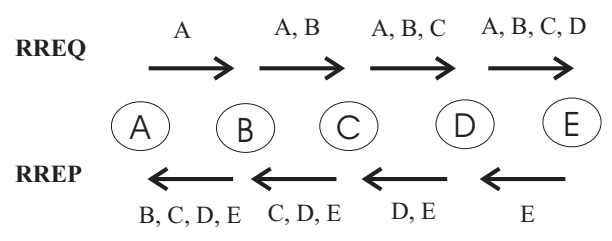

Fig. 1. Path accumulation in AODV-PA.

contained in the packet header. If a link breaks and the next node on the source route is currently not its neighbor, the node reports an error back to the source, and leaves it to the source to establish a new route. Alternatively, the node may try a different path, if it has an alternate route cached. DSR stores multiple paths per destination and does not use any expiry timers on route cache entries.

As an advantage, source routing in DSR eliminates routing tables and the aggressive caching reduces the overhead of DSR. However, there are two primary disadvantages of DSR, as found in [3]. Route reply flooding in DSR results in costly MAC layer overhead. Secondly, DSR is not scalable to large networks.

\section{PATH ACCUMUlation IN AODV}

AODV can be modified to enable path accumulation during the route discovery cycle. When the RREQ and RREP messages are generated or forwarded by the nodes in the network, each node appends its own address on these route discovery messages. Each node also updates its routing table with all the information contained in the control messages. As the RREQ messages are broadcast, each intermediate node that does not have a route to the destination forwards the RREQ packet after appending its address in the packet. Hence, at any point the RREQ packet contains a list of all the nodes traversed. Whenever a node receives a RREQ packet, it updates the route to the source node. It then checks for intermediate nodes accumulated in the path. A new entry is made in the routing table for any of the intermediate nodes, if one did not already exist. If a route entry for a node does exist, and if the hop count to any of the intermediate nodes is less than the previously known hop count to that node, the routing table entry is updated for that node. The entry is updated by retaining the previously known sequence number for that node. Note that if the node was unknown previously, the sequence number in the routing table entry is set to zero and the hop count value is obtained from the accumulated route. This conservative nature of updating the routing table along with maintaining lifetimes for each route entry helps to invalidate the stale entries and keep the route entries current, thus improving the routing accuracy of the protocol.

As the RREP message is unicast back to the source, each intermediate node forwards the RREP packet by adding its address in the packet. Hence, at any point the RREP packet contains all the previously visited nodes. Similar to the RREQ, the routing table is updated for each intermediate node visited by the RREP in addition to the destination node.

Following the guidelines of AODV, entries are also created in the precursor lists by a node forwarding a route reply back to the source. If an entry is updated to any intermediate nodes, any pending packets to that node are sent.
As an example, consider five nodes A, B, C, D and E as shown in figure 1. Node A wants to send data to node E. Since A does not have a route for $\mathrm{E}$ in its routing table, it broadcasts a route request. $\mathrm{B}$ receives the route request, updates its routing table for the reverse route to $\mathrm{A}$, and forwards the request since it also has no route to E. However, before forwarding, it appends its own address to the request. When $C$ receives the RREQ, it updates its routing table for both node $\mathrm{A}$ and $\mathrm{B}$ and appends its address to the request. Similarly, when D receives the request it updates its routing table for nodes $\mathrm{A}, \mathrm{B}$ and $\mathrm{C}$, while $\mathrm{E}$ learns about nodes A, B, C and D.

Thus, the routing table is populated and during subsequent route requests, there is higher probability of routes being present in the routing table. This should decrease the number of route discovery cycles as compared to basic AODV. This design increases the efficiency of AODV.

\section{Simulation Model}

The NS-2 network simulator was used to create a simulation environment to develop and analyze the proposed protocol (AODV-PA) and compare it with the already existing AODV and DSR on-demand ad hoc routing protocols. Simulations were run to obtain an estimate of the efficiency and the overhead cost of the three protocols.

\section{A. Traffic and mobility models}

Constant bit rate (CBR) traffic sources with 512 byte data packets are used. The source-destination pairs are spread randomly over the network. The number of sources is varied in the simulations. The packet sending rate is set to 4 packets/second. The mobility model uses the random waypoint model. Field configurations of $1000 \mathrm{~m} \times 1000 \mathrm{~m}$ field with 50 nodes and $1500 \mathrm{~m} \times 1500 \mathrm{~m}$ field with 100 nodes are used. Each node uses 802.11 with a $250 \mathrm{~m}$ transmission radius. We have kept the pause time constant at 30 seconds for all our simulation experiments. Route timeout value of $3000 \mathrm{~ms}$ is used for AODV and AODV-PA, as suggested by the AODV draft.

Simulations are run for 600 simulated seconds. Each data point represents an average of five runs with identical traffic models, but different randomly generated mobility scenarios. For fairness, identical mobility and traffic scenarios are used across protocols.

Nodes in all the three protocols maintain a send buffer of 64 packets. Each node buffers all data packets while waiting for a route. All packets (both data and routing) sent by the routing layer are queued at the interface queue until the MAC layer can transmit them. Routing packets are given higher priority than data packets in the interface queue.

\section{B. Performance Metrics}

The following metrics are used in varying scenarios to evaluate the different protocols:

1) Packet delivery ratio - This is defined as the ratio of the number of data packets received by the destinations to those sent by the CBR sources.

2) Normalized routing load - This is defined as the number of routing packets transmitted per data packet delivered at 


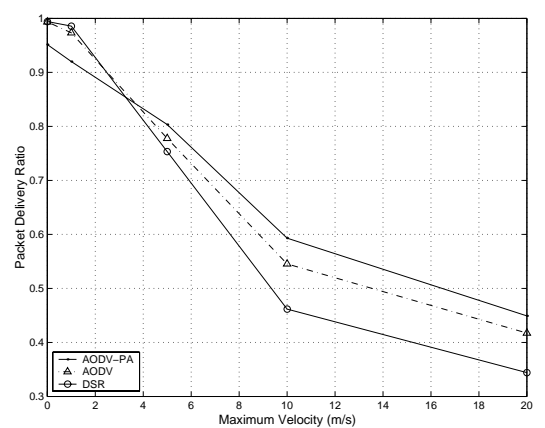

Fig. 2. Packet Delivery Ratio vs. Velocity, 20 connections.

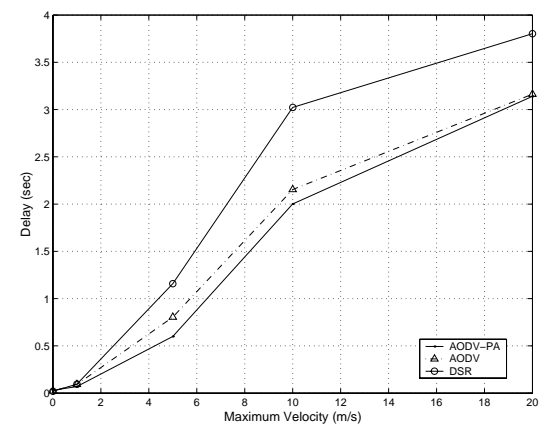

Fig. 4. Delay vs. Velocity, 20 connections.

the destination. Normalized routing load gives a measure of the efficiency of the protocol.

3) End-to-end delay of data packets - This is defined as the delay between the time at which the data packet was originated at the source and the time it reaches the destination. Data packets that get lost en route are not considered. Delays due to route discovery, queuing and retransmissions are included in the delay metric.

The metrics are measured against various mobility scenarios and with varying number of data connections.

\section{Scenarios}

A wide variety of node scenario files and CBR scenario files were generated to evaluate varying network conditions. The parameters varied in the simulations are

- Maximum velocity of the nodes

- Maximum number of data connections

- Number of nodes

Simulations are carried out by keeping the number of connections constant, and varying the velocity. 20 data sessions are modeled to study the effect of varying mobility in networks of 50 nodes. Then, the number of connections is varied from 10 to 50 in intervals of 10 for 50 nodes and from 10 to 100 for 100 nodes. While varying the number of data connections, velocity is kept at a uniform rate of $0-20 \mathrm{~m} / \mathrm{s}$.

\section{RESULTS}

\section{A. Varying Velocity}

The first experiment varies the velocity for 20 connections. The network size is 50 nodes. The results were calculated at

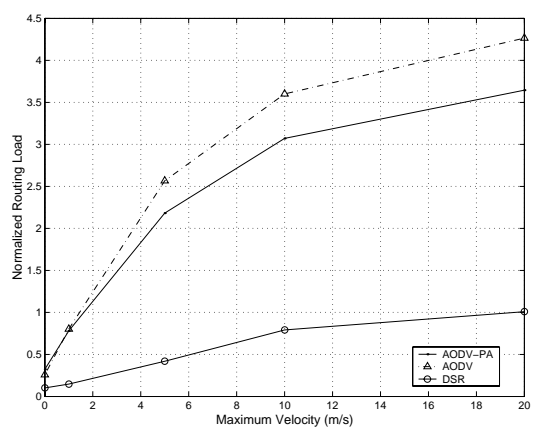

Fig. 3. Normalized Routing Load vs. Velocity, 20 connections.

constant speeds of $0,1,5,10$ and $20 \mathrm{~m} / \mathrm{s}$. The packet delivery ratio is shown in figure 2. AODV-PA has significantly higher packet delivery ratio than both AODV and DSR. The ratio of the percentage of packets delivered by AODV-PA in comparison with DSR increases from approximately 0.95 at $0 \mathrm{~m} / \mathrm{s}$ to 1.4 at $20 \mathrm{~m} / \mathrm{s}$. At $0 \mathrm{~m} / \mathrm{s}$, AODV-PA has lower packet delivery ratio value than AODV and DSR. This is because of increase in the number of packet collisions at the MAC layer.

It can be seen from figure 3 that the difference in normalized routing load between AODV-PA and AODV increases slowly with the increase in velocity. As the velocity increases, a larger percentage of nodes come within the range of each other, and the network topology information is quickly distributed. This aids in reduction of the number of route discoveries because of the additional routing information in AODV-PA. We confirmed this reduction by measuring the number of route discoveries in AODV-PA and AODV. Though not shown, it was also found that increasing the lifetime of the routes leads to a further decrease in the routing load in AODV-PA at low velocities but the increase has an adverse effect on the routing load and packet delivery ratio at moderate to high velocities. This is because routes become stale as the velocity increases, with larger lifetime values.

Figure 4 shows the effect of velocity on the average endto-end delay. Delay in DSR increases more rapidly as compared with AODV and AODV-PA as velocity increases. Delay of AODV and AODV-PA remains approximately equal.

\section{B. Varying Number of Connections}

The second experiment varies the number of connections with a random velocity of $0-20 \mathrm{~m} / \mathrm{s}$ for 50 and 100 nodes. The packet delivery ratio for AODV-PA is slightly less than AODV for fewer connections (figure 5). It then stabilizes and becomes better than AODV with the increase in the number of connections. Packet delivery ratio for DSR and AODV is similar for 10 and 20 connections. The packet delivery ratio for DSR decreases as compared to AODV-PA for higher number of connections. The ratio drops further at 100 nodes. This is because of the aggressive caching of DSR increases the number of cached routes that become stale. This is further explained in the following section.

As can be seen from figure 6, the routing load of AODV-PA decreases as compared to AODV as the number of connections increases. The routing load of AODV and AODV-PA is much 


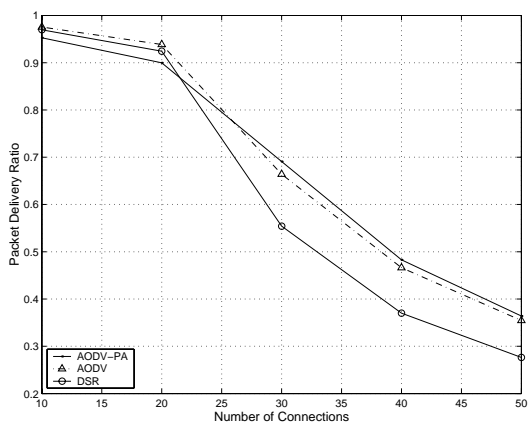

(a) 50 Nodes.

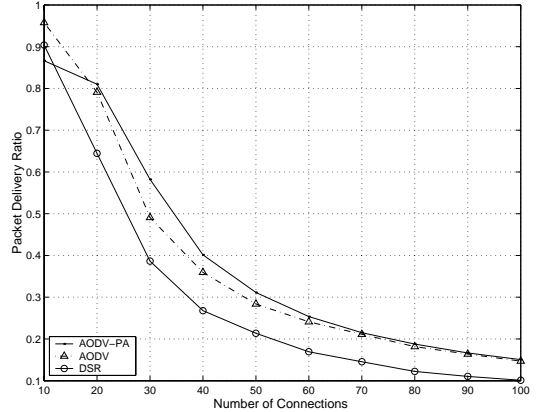

(b) 100 Nodes.

Fig. 5. Packet Delivery Ratio vs. Number of Connections.

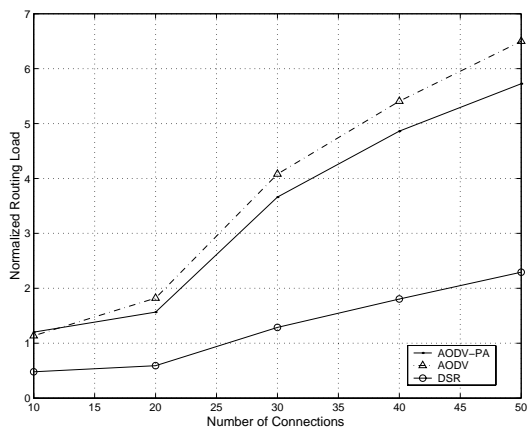

(a) 50 Nodes.

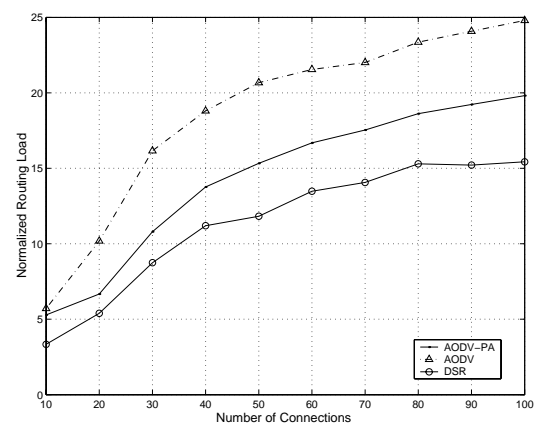

(b) 100 Nodes.

Fig. 6. Normalized Routing Load vs. Number of Connections.

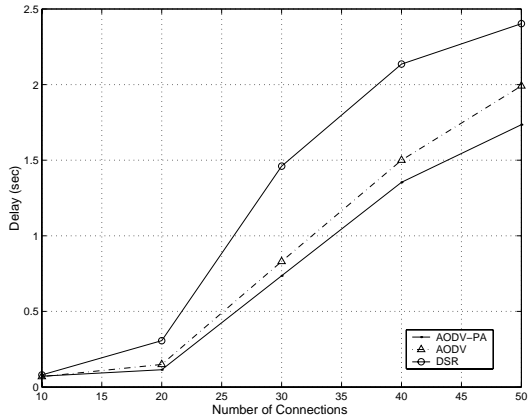

(a) 50 Nodes.

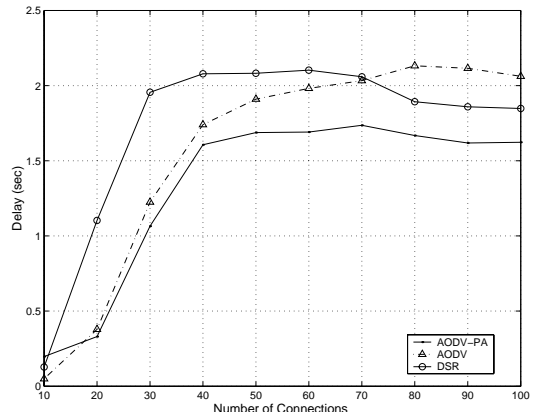

(b) 100 Nodes.

Fig. 7. Delay vs. Number of Connections.

higher than that of DSR. This is due to the aggressive caching of DSR. Even though AODV-PA performs path accumulation, it takes a conservative approach by relying on sequence numbers and route expiry timers. Therefore the number of route discoveries in AODV-PA is more than DSR and hence AODV-PA has a greater routing load. The number of routes accumulated in AODV-PA increases with the number of nodes and connections. As a result, at 50 connections, the ratio of the routing load of AODV-PA to DSR decreases from 2.5 for 50 nodes to 1.25 for 100 nodes.

Delay values for both AODV and AODV-PA are better than for DSR under all conditions (figure 7). Delay of AODV becomes greater than DSR for very high load. AODV-PA has less delay than AODV under all conditions. The difference becomes significant at large number of connections because of the decrease in the average route discovery time per packet delivered to the destination.

\section{OBSERVATIONS}

We discuss these results and compare the performance of the three protocols below. 


\section{A. Packet Delivery Ratio}

The packet delivery ratio of AODV-PA is similar to AODV under all conditions. The protocol suffers a little at fewer connections and low velocities. At low velocities, packets are dropped in AODV and AODV-PA due to packet collisions. The number of collisions increases in AODV-PA because of additional pending data packets sent by the intermediate routes during route discovery. The additional packets are sent when intermediate nodes gather routing information due to path accumulation. Because DSR does not use expiry timers, the number of stale routes increases with increase in connections and high mobility. The number of stale routes further increases with the number of nodes in the network. As a result, the packet delivery ratio of DSR decreases in these scenarios.

\section{B. Routing Load}

AODV-PA reduces the routing load as compared to AODV, particularly under high load scenarios. AODV-PA uses aggressive accumulation of the routes during its route discovery process. This increased knowledge of the network reduces the number of route discoveries in AODV-PA, which leads to a decrease in the routing load. However, the routing load of AODVPA is not as small as DSR. Even though AODV-PA uses a similar accumulation of routes as DSR, it differs in a subtle but very important manner. AODV-PA utilizes a more conservative approach than DSR by making use of expiry timers for its routing table entries. It attempts to keep the routes fresh and invalidate the stale routes. DSR does not use any timers and invalidates its routing table entries only on a link break. This helps in improving the performance of AODV-PA for application oriented metrics such as delay and packet delivery ratio by using only valid and current routes. Because stale entries may linger in DSR's cache, these routes are likely to be selected after a link break, as shown in [3].

The fact that AODV-PA performs better than AODV under moderate to high mobility scenarios might seem counter intuitive. One would expect the routes to become invalid. However, because of the use of expiry timers in AODV and AODV-PA, routes are not stale. As a result, the additional routing information due to the cached routes in AODV-PA helps to reduce the number of route discoveries as compared to that in AODV. At very high velocities, the cached routes become invalid more frequently than at moderate velocities. As a result, the difference in routing load between AODV-PA and AODV stabilizes at high velocities. The performance of AODV-PA improves with the increase in the number of nodes. This is because the number of routes accumulated during route discovery increases as the number of nodes increase.

The size of the control packets in the AODV-PA protocol is larger than that of AODV. This is compensated by the decrease in the number of routing packets in AODV-PA. Though not shown, the byte overhead (number of control packet header bytes per data byte transferred) of AODV-PA is much less than DSR and nearly equal to AODV. While the AODV-PA packets have a higher chance of collision because they are larger, the overall network overhead and energy cost to transmit the fewer packets in AODV-PA is less than that in AODV, due to the cost of acquiring the channel [4].

\section{Delay}

AODV-PA has less delay than both AODV and DSR under almost all possible scenarios. The difference is magnified under high load and moderate mobility conditions. The primary reason is that the number of route discoveries is reduced in AODVPA as compared to that in AODV. AODV-PA performs considerably better than DSR, because DSR focuses on routes with the fewest hops, while AODV-PA and AODV tend to choose the least congested route. Also, when utilizing promiscuous listening DSR has to spend time processing any control packet it receives, even if it is not the intended recipient. For 100 nodes, the average hop-count decreases with increase in the number of connections. As a result the delay curves taper-off.

\section{CONCLUSION}

This paper proposes a new protocol that modifies AODV to improve its performance. The protocol, AODV-PA, incorporates path accumulation during the route discovery process in AODV to attain extra routing information. It is evident from the results that AODV-PA improves the performance of AODV under conditions of high load and moderate to high mobility. AODV-PA also scales better than AODV in large networks. Under most conditions, AODV-PA has a higher packet delivery ratio and lower delay than DSR, though the routing load of DSR is slightly less than that of AODV-PA. The difference in the routing load of AODV-PA and DSR decreases with an increase in the load. AODV-PA can be used either as an alternative to AODV or as an optimization under moderate to high load scenarios. AODV-PA could also be suitable either if overall routing load or if application oriented metrics such as delay and packet delivery ratio are important for the ad hoc network application.

\section{ACKNOWLEDGEMENTS}

This work was supported in part by the NSF Infrastructure grant (EIA - 0080134). We would like to thank Aditya Thyagarajan for his help and the anonymous referees for their valuable comments.

\section{REFERENCES}

[1] C. E. Perkins and E. M. Royer, "Ad-hoc On-Demand Distance Vector Routing," in Proceedings of the $2^{\text {nd }}$ IEEE Workshop on Mobile Computing Systems and Applications, New Orleans, LA, February 1999, pp. 90-100.

[2] D. B. Johnson and D. A. Maltz, "Dynamic Source Routing in Ad Hoc Wireless Networks," in Mobile Computing, T. Imielinski and H. Korth, Eds. Kluwer Academic Publishers, 1996, pp. 153-181.

[3] S. R. Das, C. E. Perkins, E. M. Royer, and M. K. Marina, "Performance Comparison of Two On-Demand Routing Protocols for Ad Hoc Networks,' in IEEE Personal Communications Magazine special issue on Ad hoc Networking, February 2001, pp. 16-28.

[4] J. Broch, D. A. Maltz, D. B. Johnson, Y.-C. Hu, and J. Jetcheva, "A Performance Comparison of Multi-Hop Wireless Ad Hoc Network Routing Protocols," in Proceedings of the $4^{t h}$ International Conference on Mobile Computing and Networking, Dallas, Texas, October 1998, pp. 85-97.

[5] E. Royer and C.-K. Toh, "A Review of Current Routing Protocols for AdHoc Mobile Wireless Networks," in IEEE Personal Communications, April 1999, pp. 46-55. 